\title{
Anomalous transition magnetic moments in two-dimensional Dirac materials
}

\author{
Sanghita Sengupta, ${ }^{1}$ Madalina I. Furis, ${ }^{2,3}$ Oleg P. Sushkov, ${ }^{4}$ and Valeri N. Kotov ${ }^{3,2}$ \\ ${ }^{1}$ Institut Quantique and Département de Physique, Université de Sherbrooke, Sherbrooke, Québec, Canada J1K $2 R 1$ \\ ${ }^{2}$ Materials Science Program, University of Vermont, Burlington, Vermont 05405, USA \\ ${ }^{3}$ Department of Physics, University of Vermont, Burlington, Vermont 05405, USA \\ ${ }^{4}$ School of Physics, University of New South Wales, Sydney 2052, New South Wales, Australia
}

(Received 2 January 2020; accepted 2 July 2020; published 20 July 2020)

\begin{abstract}
We show that the magnetic response of atomically thin materials with a Dirac spectrum and spin-orbit interactions can exhibit strong dependence on electron-electron interactions. While graphene itself has a very small spin-orbit coupling, various two-dimensional (2D) compounds "beyond graphene" are good candidates to exhibit the strong interplay between spin-orbit and Coulomb interactions. Materials in this class include dichalcogenides (such as $\mathrm{MoS}_{2}$ and $\mathrm{WSe}_{2}$ ), silicene, germanene, and 2D topological insulators described by the Kane-Mele model. We present a unified theory for their in-plane magnetic field response leading to "anomalous," i.e., electron interaction dependent, transition moments. Our predictions can be potentially used to construct unique magnetic probes with high sensitivity to electron correlations.
\end{abstract}

DOI: 10.1103/PhysRevB.102.024432

\section{INTRODUCTION}

Two-dimensional quantum materials are characterized by low-energy quasiparticle excitations that can be fully described by an effective $(2+1)$-dimensional Dirac equation. Naturally, various quantum electrodynamics (QED) phenomena associated with Dirac physics manifest themselves in these quantum condensed-matter systems [1-4] even though the Dirac quasiparticles have a nonrelativistic nature and arise purely from band structure considerations.

One such astonishing feature associated with this class of materials is their magnetic response. In the presence of a magnetic field, the Dirac fermions exhibit a plethora of quantum phases which can range from anomalous quantum Hall states [5,6] to quantum holography in graphene flakes [7]. While most studies related to anomalous quantum Hall physics have been conducted within the context of massless two-dimensional (2D) Dirac fermions [5,6,8,9], recent research has elucidated similar magnetic phenomena arising in the regime of massive 2D Dirac fermions [10,11].

In this paper we explore the magnetic response of the massive 2D Dirac fermions, with a special focus on the effect of electron-electron interactions. The candidate materials for this study include (i) quantum Spin Hall (QSH) insulator states described by the Kane-Mele model [12], (ii) the atomically thin semiconductor family of transitionmetal dichalcogenides (TMDCs) $[13,14]$, and (iii) the topological insulator family of the silicene-germanene class of materials $[15,16]$.

Published by the American Physical Society under the terms of the Creative Commons Attribution 4.0 International license. Further distribution of this work must maintain attribution to the author(s) and the published article's title, journal citation, and DOI.
Our chosen materials are characterized by a gapped Dirac spectrum. In the case of QSH states described by the KaneMele model, it was shown that the symmetry-allowed spinorbit coupling (SOC) leads to an opening of the energy gap in the linear, gapless electronic dispersion of graphene [12]. This SOC thus converts the 2D semimetallic graphene into a 2D topological insulator with gapless edge modes while being insulating in the bulk. These QSH states thus allow for the generation of dissipationless spin currents and are a topic of immense interest $[12,17,18]$. However, it was also pointed out that while the SOC in graphene is of the order of $4 \mathrm{meV}$, the gap generated by it is rather small, of the order of $\sim 10^{-3} \mathrm{meV}$ $[19,20]$. One of the goals of the present work is to study in detail the in-plane magnetic response of the Kane-Mele model where we show that Coulomb interactions can have quite a significant effect and lead to an enhanced spin-flip (transition) magnetic moment.

Our theoretical approach is conceptually similar to calculations performed in relativistic QED [21,22] where Schwinger's celebrated vertex correction to the Dirac electron magnetic form factor translates into an anomalous (finestructure constant dependent) $g$ factor. Of course all materials considered in this work are nonrelativistic systems with effective Dirac quasiparticles; thus, any "anomalous" corrections to the spin response will originate from the Coulomb interaction between quasiparticles. Naturally, the results for the KaneMele model and the other 2D systems with SOC will be anisotropic since it is well known that all of them exhibit strong intrinsic spin anisotropy, with the spin $z$ component (perpendicular to the planes) conserved. This means that only in-plane magnetic fields, leading to off-diagonal (spinflip) transitions, can give rise to anomalous, i.e., Coulomb interaction dependent, transition magnetic moments. We also point out that interaction-dependent magnetic moments were recently studied for three-dimensional Dirac and Weyl insulators [23]. Compared to those systems, the spin response of 
2D materials with SOC is also, naturally, quite different, and we describe it in detail in this work.

As mentioned before, we will also extend and apply our formalism and calculations of anomalous transition magnetic moments to two other systems, which include the atomically thin TMDCs and silicene-germanene class of materials. Besides being gapped, these materials also display strong intrinsic spin-orbit coupling effects [14,24-31]. Thus, the interplay of electron-electron interactions and SOC in these systems is a topic of great interest.

The general structure of the paper is as follows: we will begin with the Kane-Mele model in Sec. II, providing the general methodology and results for the one-loop correction to the transition magnetic moment. We will then adapt and extend this formalism to TMDCs and the silicene-germanene class of materials in Secs. III and IV. Finally, we will conclude in Sec. V with an outlook that summarizes our results. We also discuss possible experimental probes for detection of the interaction effects calculated in this work.

\section{EFFECT OF COULOMB INTERACTIONS ON THE TRANSITION (SPIN-FLIP) MAGNETIC MOMENT WITHIN THE KANE-MELE MODEL}

The Kane-Mele model describes the general 2D Dirac Hamiltonian with a mass term that originates from the spinorbit coupling. This SOC renders the system gapped, and much of this section will be devoted to understanding the interplay of Coulomb interactions and the SOC in relation to the transverse magnetic response. Let us begin with the general procedure to calculate the one-loop correction to the transition moment for this model. The Hamiltonian of the Kane-Mele model [12] is

$$
H=v \sigma \cdot \mathbf{k}+\lambda \sigma_{z} s_{z}
$$

where $v$ is the Fermi velocity in the material. It is convenient, and customary in the literature, to label the spin $z$ component of the fermion as lowercase $s_{z}= \pm 1$ for spins up and down. The Pauli matrices $\hat{\sigma}_{i}$ act in pseudospin (sublattice) space, and the spin-orbit coupling is given by $\lambda$. In our derivations we choose the convenient natural units $\hbar=v=1$, unless otherwise mentioned. From the Hamiltonian we can see that the spin in the $z$ channel is always conserved. This means that interaction corrections to the diagonal (same spin) transitions are forbidden, while spin-flip transitions [caused by magnetic field in the $S_{x}$ (or $S_{y}$ ) direction] can acquire Coulomb interaction dependent components. We refer to such interaction contributions as "anomalous" spin response components.

Without loss of generality, in this and the next sections, we work in a given valley (already assumed in the above Hamiltonian). It is easy to see that the results for the spin response are valley independent (which also applies to the interaction corrections since the long-range Coulomb interaction does not mix valleys). We will also be assuming, in this and all other sections, that the system always remains an insulator (i.e., the chemical potential is in the gap).
Within the Hamiltonian of the Kane-Mele model, the dispersion relation $\varepsilon_{k}$ and eigenfunctions at momentum $k$ are given as

$$
\begin{gathered}
\varepsilon_{k}= \pm \sqrt{k^{2}+\lambda^{2}}, \\
\Psi(k)_{+}=\frac{k}{\sqrt{2} \sqrt{\varepsilon_{k}^{2}-\lambda\left|\varepsilon_{k}\right|}}\left(\frac{1}{\left(\frac{\left|\varepsilon_{k}\right|-\lambda}{\left(k_{x}-i k_{y}\right)}\right),}\right. \\
\Psi(k)_{-}=\frac{k}{\sqrt{2} \sqrt{\varepsilon_{k}^{2}+\lambda \mid \varepsilon_{k}} \mid}\left(\begin{array}{c}
1 \\
\frac{\left|\varepsilon_{k}\right|+\lambda}{\left(k_{x}-i k_{y}\right)}
\end{array}\right) .
\end{gathered}
$$

The wave functions $\Psi(k)_{s}$ are labeled by the spin index $s_{z}=$ $s= \pm 1$.

Next, we consider coupling to a uniform in-plane magnetic field of the form $B_{x} S_{x}$, with the coupling constant given by the $g$ factor times the effective Bohr magneton set to 1 for convenience, $g \mu_{B}=1$. We define a quantity we call the bare transition magnetic moment as $\mu=2\left\langle\downarrow\left|S_{x}\right| \uparrow\right\rangle$. Here, the (normalized) spin-up state is a product of the pseudospin and spin wave functions: $|\uparrow\rangle=\Psi(k)_{+} \chi_{+}$, where $\chi_{+}=\left(\begin{array}{l}1 \\ 0\end{array}\right)$ is the spin-up spinor in spin space. Similarly, $|\downarrow\rangle=\Psi(k)_{-} \chi_{-}$, $\chi_{-}=\left(\begin{array}{l}0 \\ 1\end{array}\right)$. From the usual spin- $1 / 2$ algebra we have $2 S_{x} \chi_{+}=\chi_{-}$.

Using the above wave functions, we calculate the bare transition moment for this model:

$$
\begin{aligned}
\mu & =2\left\langle\downarrow\left|S_{x}\right| \uparrow\right\rangle=\left[\chi_{-}^{\dagger}\left(2 S_{x}\right) \chi_{+}\right]\left[\Psi(k)_{-}^{\dagger} \Psi(k)_{+}\right] \\
& =\Psi(k)_{-}^{\dagger} \Psi(k)_{+}=\frac{k}{\sqrt{k^{2}+\lambda^{2}}} .
\end{aligned}
$$

From now on we will use the shorthand notation $|\uparrow\rangle,|\downarrow\rangle$ in all calculations in this section as well as for the models considered in subsequent sections.

We proceed to calculate the effect of electron-electron (Coulomb) interactions on the transition magnetic moment. Basic Feynman diagrams for the bare and one-loop (vertex) corrections are given in Fig. 1. Invoking Feynman rules, we will write an analytic expression corresponding to the vertex function given in the right panel of Fig. 1.

Therefore, the one-loop Coulomb interaction correction to the magnetic moment (for $q \rightarrow 0$ ) is given as

$$
\begin{aligned}
\delta \mu= & 2 \sum_{p} i \int \frac{\mathrm{d} \omega}{2 \pi}\left\langle\downarrow\left|G^{s=-1}(p, \omega) S_{x} G^{s=+1}(p+q, \omega)\right| \uparrow\right\rangle \\
& \times V(|\mathbf{p}-\mathbf{k}|),
\end{aligned}
$$

where $V(\mathbf{p})=\left(2 \pi e^{2} / p\right)$ is the Coulomb interaction and the corresponding Green's function for this model is

$$
G(\mathbf{k}, \omega)=\frac{\omega+\left(\sigma \cdot \mathbf{k}+\lambda \sigma_{z} s_{z}\right)}{\omega^{2}-\varepsilon_{k}^{2}+i \eta} .
$$

Using the above equations along with the corresponding wave functions [Eqs. (3) and (4)], we derive an expression for the one-loop interaction correction,

$$
\delta \mu=\frac{k}{\sqrt{k^{2}+\lambda^{2}}} \alpha \mathcal{W}(k / \lambda),
$$




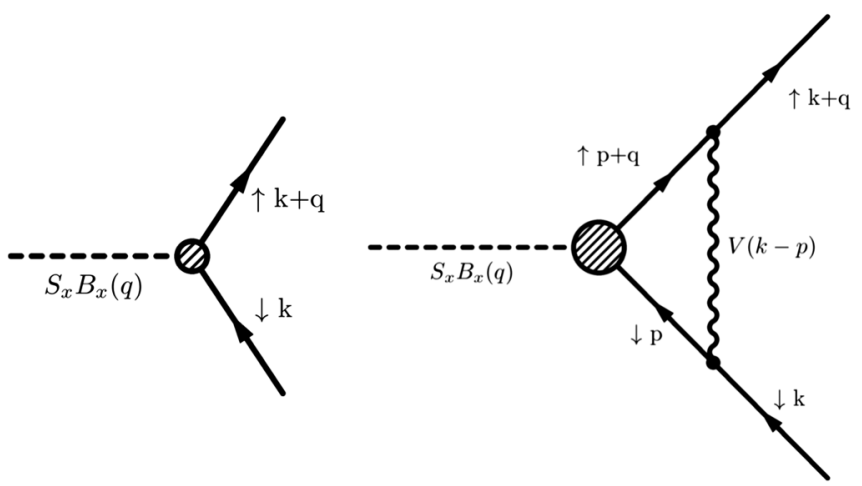

FIG. 1. Left: Feynman diagram for the bare transition moment with uniform (zero momentum, $q \rightarrow 0$ ) in-plane magnetic field $B_{x}$, corresponding to field coupling of the form $B_{x}(q \rightarrow 0) S_{x}$. We set the field coupling prefactor $g \mu_{B}=1$ in this field direction for simplicity (it is known that the $g$ factor can be strongly material dependent and should be restored when comparison with experiment is made). Right: Vertex diagram associated with the one-loop Coulomb interaction correction shown by the wiggly line $\mathrm{V}(\mathbf{p})=2 \pi e^{2} / p$.

where we have defined

$$
\alpha \mathcal{W}(k / \lambda)=\frac{\lambda^{2}}{2} \int \frac{d^{2} p}{(2 \pi)^{2}} \frac{V(|\mathbf{p}-\mathbf{k}|)}{\left|\varepsilon_{p}\right|^{3}}\left(1-\frac{\mathbf{p} \cdot \mathbf{k}}{k^{2}}\right),
$$

with $\alpha=e^{2} / \epsilon \hbar v$ being the effective fine-structure constant representing the strength of Coulomb interactions and $\epsilon$ being the dielectric constant.

The variation of the correction function $\mathcal{W}(k / \lambda)$ with the dimensionless band momenta $(k / \lambda)$ is shown in the top panel of Fig. 2. The Coulomb interaction correction peaks at $k=0$ and thereafter decays with the increase in band momenta.

Using Eqs. (5), (8), and (9), we write the total transition moment as

$$
\begin{aligned}
\mu+\delta \mu= & \frac{k}{\sqrt{k^{2}+\lambda^{2}}}\left[1+\frac{\lambda^{2}}{2} \int \frac{d^{2} p}{(2 \pi)^{2}} \frac{V(|\mathbf{p}-\mathbf{k}|)}{\left|\varepsilon_{p}\right|^{3}}\right. \\
& \left.\times\left(1-\frac{\mathbf{p} \cdot \mathbf{k}}{k^{2}}\right)\right] .
\end{aligned}
$$

To display the effects of the Coulomb interaction correction, we show the dependence of the total transition moment $\mu+$ $\delta \mu$ with the dimensionless band momenta for various values of the coupling $\alpha$ in the bottom panel of Fig. 2 .

The maximum value of $\alpha=2.2$ can, in principle, be achieved in suspended samples, while additional effects leading to coupling constant renormalization due to self-consistent screening and/or substrate effects should also be taken into account. All of these lead to a decrease in the effective coupling. First, the presence of a substrate with dielectric constant $\kappa$ will reduce the Coulomb coupling $\alpha$ via $\alpha \rightarrow$ $\alpha / \epsilon$, where $\epsilon=(1+\kappa) / 2$, assuming the $2 \mathrm{D}$ material is on a substrate with air on the other side. For example, the dielectric constant of the commonly used $\mathrm{SiO}_{2}$ is $\kappa \approx 4$, leading to a decrease of $\alpha$ by a factor of 2.5. Second, due to the electron polarization in the $2 \mathrm{D}$ material, the Coulomb interaction is screened, which can be taken into account self-consistently within the usual random-phase approximation (RPA) scheme. The effective Coulomb interaction is obtained by the simple
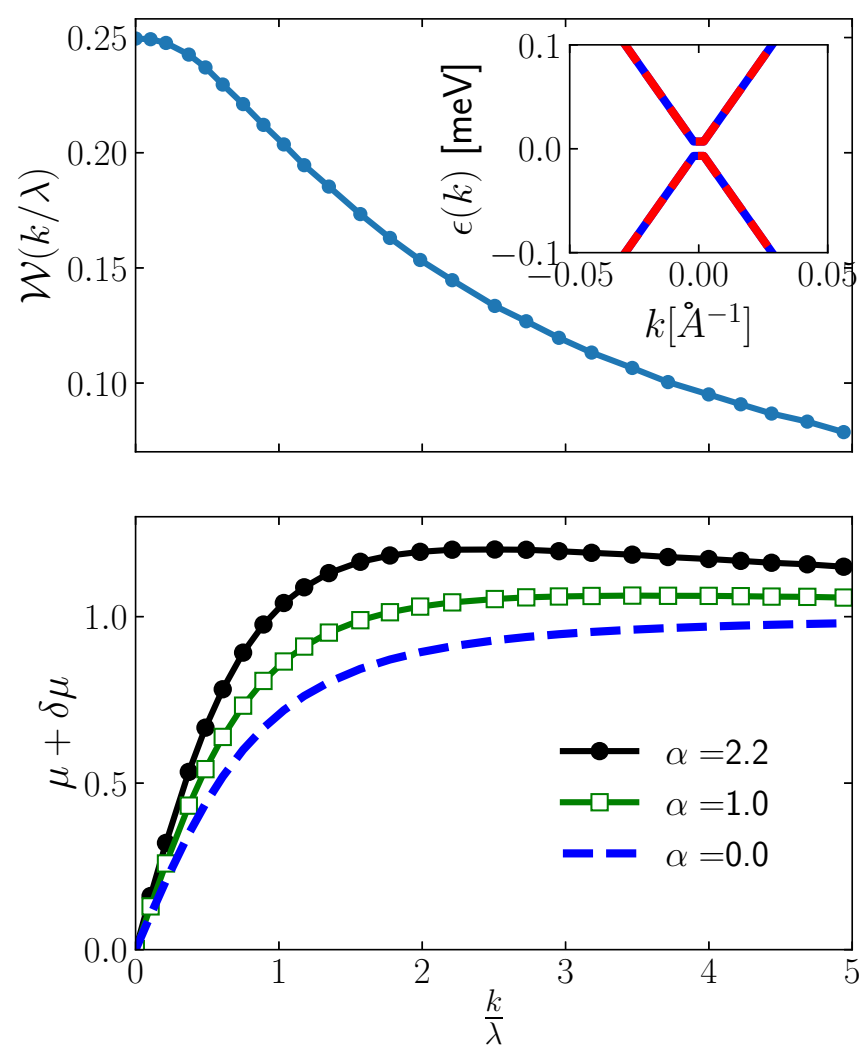

FIG. 2. Top: Variation of the correction function $\mathcal{W}(k / \lambda)$ with rescaled momentum $k / \lambda$. The magnitude of the correction is large and maximum at $k=0$. Inset: Low-energy band structure for the Kane-Mele model. Bands are spin degenerate with a gap that is generated by the spin-orbit interaction $\lambda=1 \mu \mathrm{eV}$. Bottom: Variation of the total spin-flip transition moment, $\mu+\delta \mu=\frac{k}{\sqrt{k^{2}+\lambda^{2}}}[1+$ $\alpha \mathcal{W}(k / \lambda)$ ], with rescaled momentum $(k / \lambda)$. With the increase in $\alpha$ we see an enhancement in the total transition moment. We relate this increase to the enlarged correction effects from Coulomb interactions.

replacement $V(k) \rightarrow V(k) /[1-V(k) \Pi(k)]$. In this way the results become reliable even in the regime of strong bare coupling (e.g., $\alpha=2.2$ ). We present results for static screening, which involves the static polarization function $\Pi(k, \omega=0) \equiv$ $\Pi(k)$ for a material with a gapped 2D Dirac spectrum [32,33], appropriate for the Kane-Mele model:

$$
\Pi(k)=-\frac{1}{\pi}\left(\frac{\lambda}{v^{2}}\right)-\frac{k}{2 \pi v}\left[1-\frac{4 \lambda^{2}}{v^{2} k^{2}}\right] \tan ^{-1}\left(\frac{v k}{2 \lambda}\right) .
$$

When we incorporate the effects of the gapped polarization, the total transition magnetic moment transforms to

$$
\begin{aligned}
\mu+\delta \mu= & \frac{k}{\sqrt{k^{2}+\lambda^{2}}}\left[1+\frac{\lambda^{2}}{2} \int \frac{d^{2} p}{(2 \pi)^{2}} \frac{V(|\mathbf{p}-\mathbf{k}|)}{\left|\varepsilon_{p}\right|^{3}}\right. \\
& \left.\times \frac{1}{[1-V(|\mathbf{p}-\mathbf{k}|) \Pi(|\mathbf{p}-\mathbf{k}|)]}\left(1-\frac{\mathbf{p} \cdot \mathbf{k}}{k^{2}}\right)\right],
\end{aligned}
$$

where we have used again $v=1$.

Within the RPA, assuming a suspended sample, the correction function and the total transition magnetic moment are 

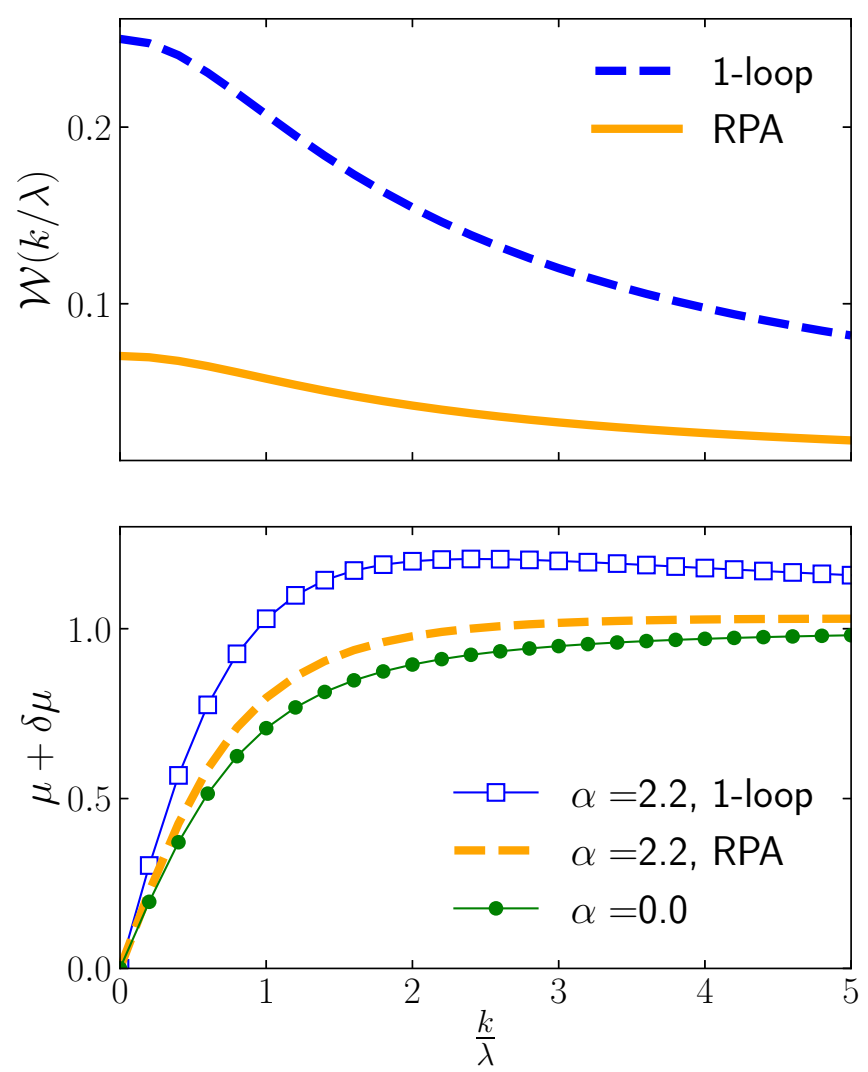

FIG. 3. Top: A comparison of the correction function $\mathcal{W}(k / \lambda)$ with rescaled momentum $k / \lambda$ for the one-loop case and the RPA. The magnitude of the correction is seen to decrease with the inclusion of self-consistent screening. Bottom: Comparative plots of the variation of the spin-flip transition moment, $\mu+\delta \mu$, with rescaled momentum $(k / \lambda)$ for the suspended case within the formalism of one-loop, RPA, and no-Coulomb correction effects.

shown in Fig. 3. It is evident that self-consistent screening further decreases the correction function, as expected. This decrease is also manifested in the decrease of the total transition magnetic moment.

In the next section we extend this formalism to calculate the one-loop Coulomb interaction correction for the transition moment in atomically thin dichalcogenides.

\section{ANOMALOUS TRANSITION MOMENT IN THE ATOMICALLY THIN FAMILY OF DICHALCOGENIDES}

In contrast to the Kane-Mele model, the atomically thin TMDCs display a large spin-independent gap (approximately of the order of a few eV) which originates from the broken inversion symmetry of the sublattice of these systems [14,24]. Along with a large spin-independent gap which we refer to as $\Delta$, these materials also display strong intrinsic spinorbit coupling arising from the admixture of the $d$ orbitals of the transition metals [24]. In this section, we will probe the Coulomb interaction effect on the SOC-induced magnetic moment of these class of materials. Our procedure will be the same as before.
The effective low-energy Hamiltonian associated with the monolayer TMDCs [14]

$$
H=\sigma \cdot \mathbf{k}+(\Delta / 2) \sigma_{z}-(\lambda / 2)\left(\sigma_{z}-1\right) s_{z} .
$$

Here, $\Delta$ is the spin-independent gap, and $\lambda$ is the spin-orbit coupling. The model parameters for $\mathrm{MoS}_{2}$ are $\Delta \approx 1.66 \mathrm{eV}$, $2 \lambda \approx 0.15 \mathrm{eV}$; for $\mathrm{WS}_{2}$ they are $\Delta \approx 1.79 \mathrm{eV}, 2 \lambda \approx 0.43 \mathrm{eV}$, and for $\mathrm{WSe}_{2}$ they are $\Delta \approx 1.6 \mathrm{eV}, 2 \lambda \approx 0.46 \mathrm{eV}[14,24]$, which clearly indicate that the family of TMDCs can be classified by a regime in which the spin-independent gap is much larger than the spin-orbit coupling,

$$
\Delta / \lambda \gg 1 \text {. }
$$

The exact wave functions at momentum $k$ for this class of materials are written as

$$
\Psi(k)_{n}^{s}=\frac{k}{\sqrt{k^{2}+\left(E_{k, n}^{s}\right)^{2}}}\left(\begin{array}{c}
1 \\
E_{k, n}^{s} /\left(k_{x}-i k_{y}\right)
\end{array}\right), \quad n=1,2,
$$

where $s$ is the spin index and $n=1$ and $n=2$ label the conduction band and valence band, respectively. Here, we have defined $E_{k, n}^{s}$ as the quantities

$$
E_{k, n}^{s}=\varepsilon_{k, n}^{s}-\Delta / 2, \quad s=s_{z}= \pm 1 .
$$

$\varepsilon_{k, n}^{s}$ represent the eigenenergies:

$$
\begin{gathered}
\varepsilon_{k, 1}^{s}=\lambda s / 2+\varepsilon_{k}^{s}>0, \quad n=1, \\
\varepsilon_{k, 2}^{s}=\lambda s / 2-\varepsilon_{k}^{s}<0, \quad n=2,
\end{gathered}
$$

with $\varepsilon_{k}^{s}$ appropriately defined as

$$
\varepsilon_{k}^{s} \equiv+\sqrt{k^{2}+\left[(\Delta-\lambda s)^{2} / 4\right]} .
$$

In the inset of the top panel of Fig. 4, we show the lowenergy band structure for this group of materials corresponding to Eq. (19). These bands are nondegenerate, showing spin inversion with a large spin-independent gap.

The bare transition moment is calculated using the wave functions for the conduction band [given in Eq. (15)], leading to

$$
\mu=2\left\langle\downarrow\left|S_{x}\right| \uparrow\right\rangle=\frac{k^{2}+E_{k, 1}^{+} E_{k, 1}^{-}}{\sqrt{\left(k^{2}+\left[E_{k, 1}^{+}\right]^{2}\right)\left(k^{2}+\left[E_{k, 1}^{-}\right]^{2}\right)}} .
$$

For the correction to the bare transition moment we will use the vertex function and Eq. (6). The Green's function for this model is

$$
\begin{aligned}
G^{s}(p, \omega)= & \frac{1}{2 \varepsilon_{p}^{s}}\left[\frac{\varepsilon_{p}^{s}+\sigma \cdot \mathbf{p}+\sigma_{z}(\Delta-\lambda s) / 2}{\omega-\varepsilon_{p, 1}^{s}+i \eta}\right. \\
& \left.-\frac{-\varepsilon_{p}^{s}+\sigma \cdot \mathbf{p}+\sigma_{z}(\Delta-\lambda s) / 2}{\omega-\varepsilon_{p, 2}^{s}-i \eta}\right] .
\end{aligned}
$$

Using the above Green's function, we first perform the frequency integral in Eq. (6) with the result

$$
\begin{aligned}
i \int \frac{d \omega}{2 \pi}\left[G^{-} G^{+}\right] \approx & \frac{1}{4 \varepsilon_{p}^{+} \varepsilon_{p}^{-}} \frac{1}{\left[\lambda^{2}-\left(\varepsilon_{p}^{-}+\varepsilon_{p}^{+}\right)^{2}\right]}\left[\lambda^{2} \frac{\Delta}{\varepsilon_{p}}(\sigma \cdot \mathbf{p})\right. \\
& \left.-2 \lambda^{2} \frac{p^{2}}{\varepsilon_{p}}\left(\sigma_{z}+1\right)-4 \lambda \varepsilon_{p}(\sigma \cdot \mathbf{p}) \sigma_{z}\right] .
\end{aligned}
$$



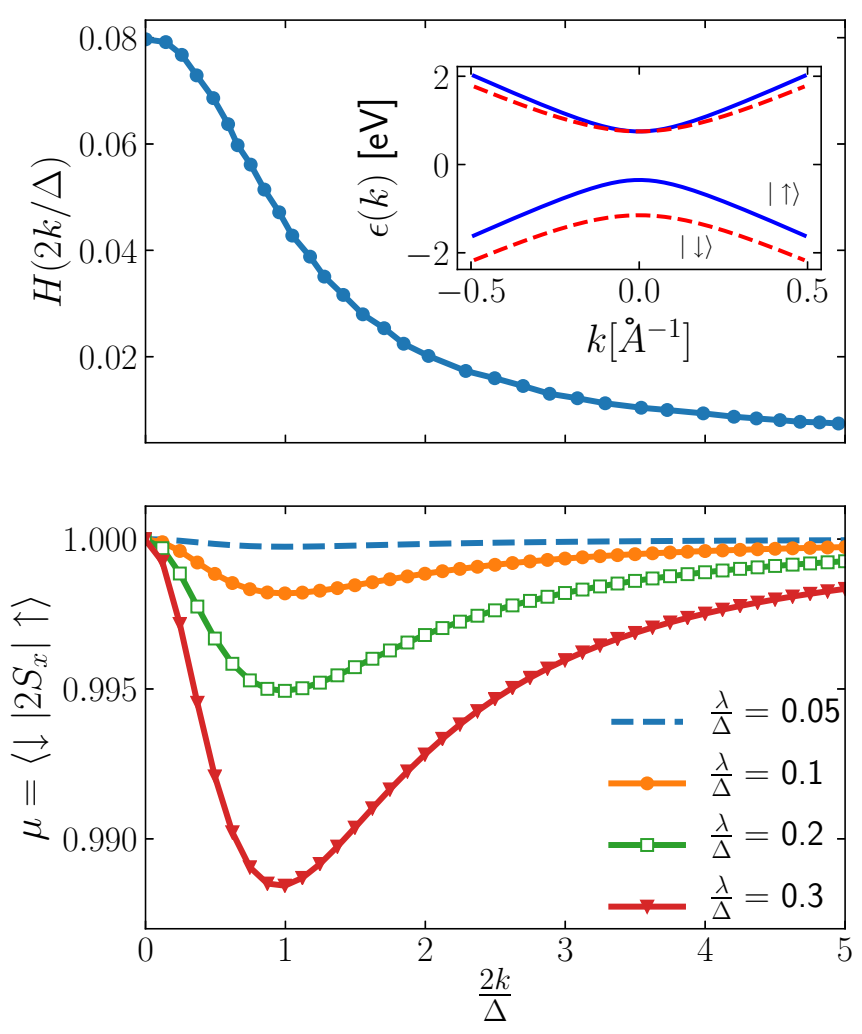

FIG. 4. Top: Correction function $H(2 k / \lambda)$ for the dichalcogenides. Here, we have used the rescaled momentum: $2 k / \Delta$. As can be seen, at $2 k / \Delta=0$, the value of the correction function $H(2 k / \Delta)$ is very small $(\sim 0.08$.) The inset in the top panel shows the corresponding dispersion relation for the dichalcogenides. The conduction bands are degenerate at $\mathrm{k}=0$ and are also seen to undergo a band inversion. Bottom: Bare transition moment for the dichalcogenides for various values of $\lambda / \Delta$. For TMDCs with the relevant value of $\lambda / \Delta \lesssim 0.15$, we see that the value of $\mu$ is almost a constant $\approx 1$ and shows negligible variation with the momentum.

Here, we have expanded the numerator up to $O\left[\lambda^{2}\right]$. The prefactors of Eq. (22) given by the energy denominators can be taken at $\lambda=0$ because their expansion starts from a constant and the next order is $O\left[\lambda^{2}\right]$. Following Eq. (6), the interaction correction to the transition moment is derived by taking the expectation value of the above equation with respect to the wave functions $\Psi(k)_{1}^{ \pm}$[Eq. (15)],

$$
\delta \mu=\sum_{\mathbf{p}} V(k-p) \frac{(-1)}{16 \varepsilon_{p}^{4}} \lambda^{2} \frac{2}{k^{2}+E_{k}^{2}} \Gamma(p, k),
$$

where the function $\Gamma(p, k)$ has been calculated as

$$
\begin{aligned}
\Gamma(p, k)= & \frac{1}{\varepsilon_{p}}\left\{\Delta E_{k}(\mathbf{k} \cdot \mathbf{p})-2 p^{2} k^{2}\right\} \\
& +2 \varepsilon_{p}(\mathbf{k} \cdot \mathbf{p})\left(1-\frac{\Delta}{2 \varepsilon_{k}}\right) .
\end{aligned}
$$

In the above expression, we have used the following definitions:

$$
E_{k}=\varepsilon_{k}-\Delta / 2, \quad \varepsilon_{k} \equiv+\sqrt{k^{2}+\left[\Delta^{2} / 4\right]} .
$$

Finally, we derive the total transition moment as the sum of the bare [Eq. (20)] and the Coulomb interaction dependent spin-flip transition moments [Eq. (23)],

$$
\mu+\delta \mu=\left\langle\downarrow\left|2 S_{x}\right| \uparrow\right\rangle\left[1+\alpha(2 \lambda / \Delta)^{2} H(2 k / \Delta)\right],
$$

where the correction term is conveniently written as

$$
\delta \mu=\left\langle\downarrow\left|2 S_{x}\right| \uparrow\right\rangle \alpha(2 \lambda / \Delta)^{2} H(2 k / \Delta),
$$

with the function $H(2 k / \Delta)$, which can be easily evaluated using Eqs. (23) and (24).

In the top panel of Fig. 4 we show the variation of the Coulomb interaction correction function $H(2 k / \Delta)$ for $\alpha=$ 4.095 with respect to the dimensionless band momenta. We observe that the magnitude of this correction is very small for this class of materials with little to no variation. Thus, the effect of the Coulomb interaction correction is the least on the spin-flip transition moment in this class of materials. This can be understood from the fact that the spin-independent gap for these materials overwhelms the contribution from the spin-orbit coupling term. Hence, this class of materials does not offer the unique tunability of the Coulomb interaction dependent effect of the spin-flip transition moment, in the sense that the interaction corrections are negligibly small for all reasonable values of $\alpha$. Additionally, from Eq. (12), which takes into account effects beyond one loop within the RPA self-consistency for the Kane-Mele model, we concluded that the Coulomb interaction correction effects decreased further. Similar RPA calculations can be performed for this class of materials; however, due to the intrinsic smallness of the one-loop results in this case, the RPA formalism leads to only a small additional decrease of the already small correction effect.

In the next section, we will derive the anomalous transition moment for the silicene-germanene class of materials.

\section{EFFECT OF COULOMB INTERACTIONS ON TRANSITION MAGNETIC MOMENTS IN THE SILICENE-GERMANENE CLASS OF MATERIALS}

An application of transverse electric field along the staggered sublattices of this class of materials causes the lowenergy band structure to evolve from a topological insulator (TI) to a bulk insulator (BI) via a valley spin-polarized metal (VSPM) state [25,26,34-36]. In this section, we will first summarize the low-energy band structure of this class of materials and show that the evolution of the low-energy band structure from a TI to a BI via a VSPM state can also be attained with proper tuning of the dimensionless parameter which represents the ratio of the spin-independent gap to the spin-orbit coupling $(\Delta / \lambda)$. This class of materials thus open up the possibility to explore the Coulomb interaction correction for a large parameter regime.

The Hamiltonian of this class of materials $[25,26,35]$ is

$$
H=v \sigma \cdot \mathbf{k}+\frac{\left(\Delta-\lambda s_{z}\right)}{2} \sigma_{z} .
$$



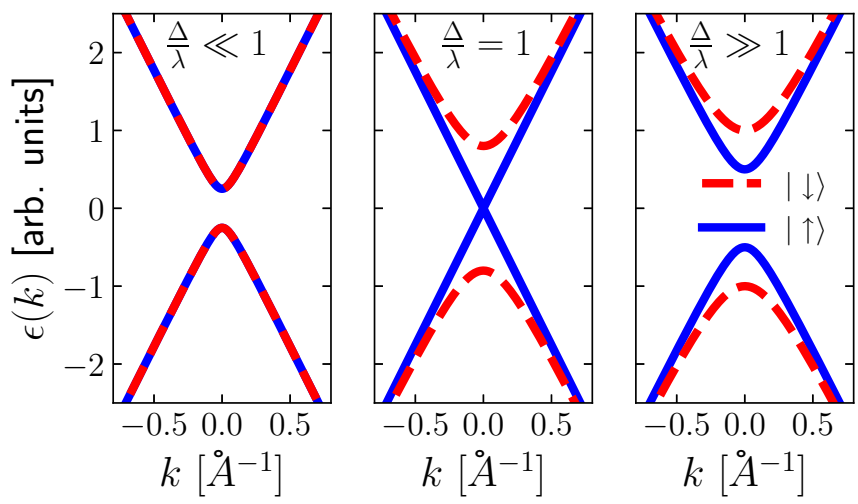

FIG. 5. For the purposes of illustration we plot the evolution of energy dispersion curves for silicene-germanene-type materials. With the proper tuning of the ratio of the spin-independent gap to spin-orbit coupling $(\Delta / \lambda)$, the band structure shows a transition from a topological insulator $(\Delta \ll \lambda)$ to a band insulator $(\Delta \gg \lambda)$ via the quantum critical VSPM state $(\Delta=\lambda)$. Subsequent removal of the spin degeneracy is also observed for the bulk insulator regime $(\Delta \gg \lambda)$.

The exact wave functions at momentum $k$ for the Hamiltonian given by Eq. (28)

$$
\Psi(k)_{n}^{s}=\frac{k}{\sqrt{k^{2}+\left(E_{k, n}^{s}\right)^{2}}}\left(\begin{array}{c}
1 \\
E_{k, n}^{s} /\left(k_{x}-i k_{y}\right)
\end{array}\right), \quad n=1,2,
$$

where $n=1$ labels the conduction band and $n=2$ labels the valence band. We have defined $E_{k, n}^{s}$ as

$$
E_{k, n}^{s}=\varepsilon_{k, n}^{s}-\frac{(\Delta-\lambda s)}{2}, \quad s=s_{z}= \pm 1,
$$

where the eigenenergies associated with the conduction and valence bands are given by $\varepsilon_{k, n}^{s}$ :

$$
\begin{gathered}
\varepsilon_{k, 1}^{s}=\varepsilon_{k}^{s}>0, \quad n=1, \\
\varepsilon_{k, 2}^{s}=-\varepsilon_{k}^{s}<0, \quad n=2,
\end{gathered}
$$

and we use the definition

$$
\varepsilon_{k}^{s} \equiv+\sqrt{k^{2}+\left[(\Delta-\lambda s)^{2} / 4\right]}
$$

Figure 5 shows the low-energy band structure corresponding to Eq. (33) plotted for the three different values $(\Delta / \lambda) \ll$ $1,(\Delta / \lambda)=1$, and $(\Delta / \lambda) \gg 1$. As can be seen, the three different cases corresponding to different values of $(\Delta / \lambda)$ are consistent with the TI, VSPM, and a BI state. General values of the spin-orbit coupling for these class of materials are in the range of $\lambda \approx 3-40 \mathrm{meV}[37,38]$. Next, we derive the expression for the bare transition moment using the conduction band wave functions given by Eq. (29).

From Eq. (29), we can write the corresponding conduction band ( $n=1)$ wave functions $|\uparrow\rangle$ and $|\downarrow\rangle$ as

$$
\begin{aligned}
& |\uparrow\rangle=\Psi(k)_{1}^{+}=\frac{k}{\sqrt{k^{2}+\left(E_{k, 1}^{+}\right)^{2}}}\left(\begin{array}{c}
1 \\
E_{k, 1}^{+} /\left(k_{x}-i k_{y}\right)
\end{array}\right), \\
& |\downarrow\rangle=\Psi(k)_{1}^{-}=\frac{k}{\sqrt{k^{2}+\left(E_{k, 1}^{-}\right)^{2}}}\left(\begin{array}{c}
1 \\
E_{k, 1}^{-} /\left(k_{x}-i k_{y}\right)
\end{array}\right) .
\end{aligned}
$$
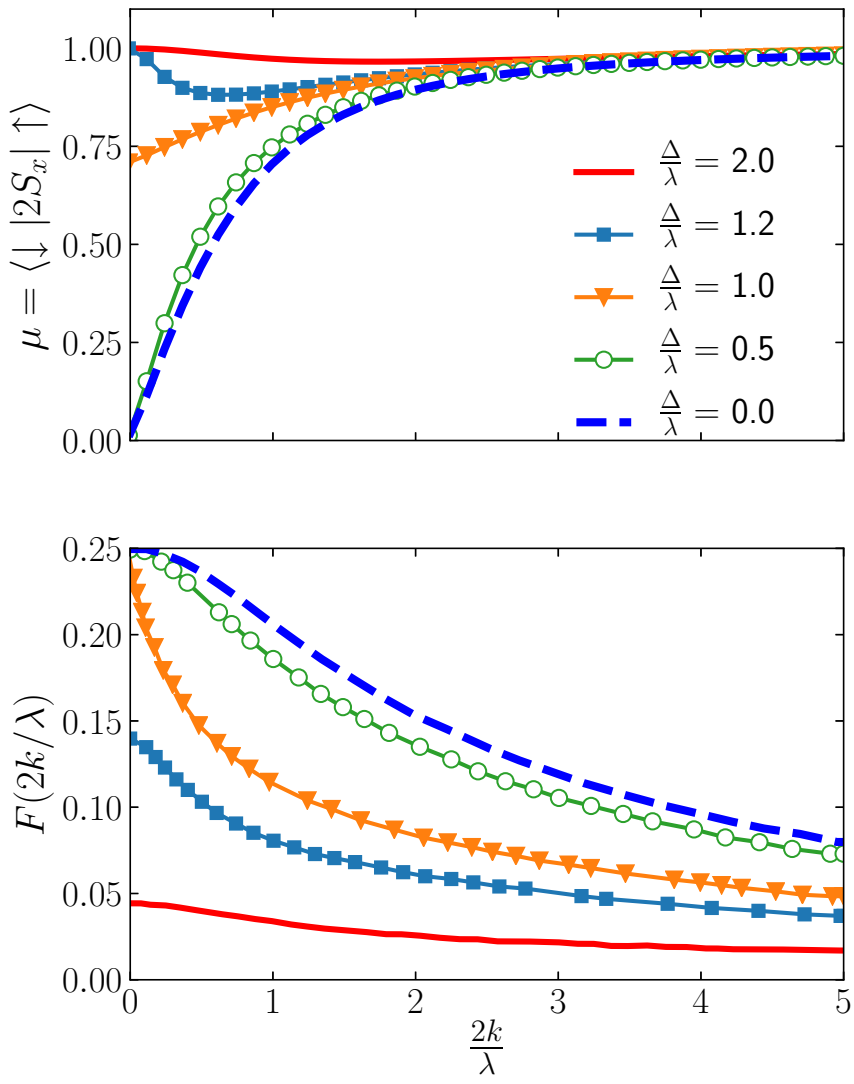

FIG. 6. Top: Variation of the bare transition moment $\mu=$ $\left\langle\uparrow\left|2 S_{x}\right| \downarrow\right\rangle$ with the rescaled momentum $2 k / \lambda$ for several values of $\Delta / \lambda$. As the coupling parameter $\Delta / \lambda$ increases, the system makes a transition from TI to BI via the VSPM state $(\Delta / \lambda=1)$. Bottom: Variation of the correction function $F(2 k / \lambda)$ with $2 k / \lambda$ for various values of coupling $0<\Delta / \lambda<2$. The correction term is seen to be large for the topological insulators $(\Delta / \lambda \ll 1)$ compared to the $\operatorname{VSPM}(\Delta / \lambda=1)$ or bulk insulator states $(\Delta / \lambda \gg 1)$.

Using Eqs. (34) and (35), we derive an expression for the band momentum dependent bare transition moment,

$$
\mu=2\left\langle\downarrow\left|S_{x}\right| \uparrow\right\rangle=\frac{k^{2}+E_{k, 1}^{+} E_{k, 1}^{-}}{\sqrt{\left(k^{2}+\left[E_{k, 1}^{+}\right]^{2}\right)\left(k^{2}+\left[E_{k, 1}^{-}\right]^{2}\right)}} .
$$

In the top panel of Fig. 6, we show the variation of the bare transition moment $\mu \equiv\left\langle\downarrow\left|2 S_{x}\right| \uparrow\right\rangle$ with a dimensionless rescaled momentum $(2 k / \lambda)$ for various values of $(\Delta / \lambda)$. The overall variation of the spin-flip transition with momentum for different values of the spin-independent gap can be intuitively understood in the following way. At $\Delta / \lambda=0$, which corresponds to the Kane-Mele model, the system is stiff in the spin $z$ direction as the term proportional to $s_{z}$ favors ordering, and thus, a transverse field at zero momentum (uniform field) cannot cause spin flip, while at finite band momentum this becomes possible due to the presence of the kinetic energy term. In the opposite extreme, $\Delta / \lambda \gg 1$, a spin flip can be achieved effortlessly as the $s_{z}$ term can be neglected.

Finally, we turn to the interaction corrections. The expression for the Green's function $G(\mathbf{k}, \omega)$ corresponding to the 
Hamiltonian of Eq. (28) reads

$$
\begin{aligned}
G(\mathbf{k}, \omega)= & \frac{1}{2 \varepsilon_{k}^{s}}\left[\frac{\varepsilon_{k}^{s}+\sigma \cdot \mathbf{k}+\left(\frac{\Delta-\lambda s_{z}}{2}\right) \sigma_{z}}{\left(\omega-\varepsilon_{k}^{s}+i \eta\right)}\right. \\
& \left.+\frac{\varepsilon_{k}^{s}-\sigma \cdot \mathbf{k}-\left(\frac{\Delta-\lambda s_{z}}{2}\right) \sigma_{z}}{\left(\omega+\varepsilon_{k}^{s}-i \eta\right)}\right] .
\end{aligned}
$$

We evaluate the frequency integral in Eq. (6) by using the Green's function expression from Eq. (37), resulting in

$$
\begin{aligned}
i \int \frac{\mathrm{d} \omega}{2 \pi}\left[G^{-} G^{+}\right]= & \frac{1}{2 \varepsilon_{p}^{-} \varepsilon_{p}^{+}}\left\{\frac{1}{\left(\varepsilon_{p}^{+}+\varepsilon_{p}^{-}\right)}\right\}\left[\varepsilon_{p}^{-} \varepsilon_{p}^{+}-(\sigma \cdot \mathbf{p})^{2}\right. \\
& \left.+\left(\frac{\lambda^{2}-\Delta^{2}}{4}\right)+\lambda(\sigma \cdot \mathbf{p}) \sigma_{z}\right]
\end{aligned}
$$

We substitute the above equation along with the conduction band wave functions given by Eqs. (34) and (35) in Eq. (6) to derive the expression for the correction term:

$$
\begin{aligned}
\delta \mu= & \left\langle\downarrow\left|2 S_{x}\right| \uparrow\right\rangle \sum_{\mathbf{p}} \frac{V(|\mathbf{p}-\mathbf{k}|)}{2 \varepsilon_{p}^{+} \varepsilon_{p}^{-}\left(\varepsilon_{p}^{+}+\varepsilon_{p}^{-}\right)}\left[\varepsilon_{p}^{+} \varepsilon_{p}^{-}-p^{2}\right. \\
& \left.+\left(\frac{\lambda^{2}-\Delta^{2}}{4}\right)+\lambda(\mathbf{p} \cdot \mathbf{k})\left\{\frac{\varepsilon_{k}^{-}-\varepsilon_{k}^{+}-\lambda}{k^{2}+E_{k, 1}^{+} E_{k, 1}^{-}}\right\}\right] \\
= & \left\langle\downarrow\left|2 S_{x}\right| \uparrow\right\rangle \alpha F(2 k / \lambda, \Delta / \lambda) .
\end{aligned}
$$

The function $F(2 k / \lambda, \Delta / \lambda)$ quantifies the Coulomb interaction correction effects, and we have defined it as

$$
\alpha F(2 k / \lambda, \Delta / \lambda) \equiv \sum_{\mathbf{p}} \frac{V(|\mathbf{p}-\mathbf{k}|)}{2 \varepsilon_{p}^{+} \varepsilon_{p}^{-}\left(\varepsilon_{p}^{+}+\varepsilon_{p}^{-}\right)}\left[\varepsilon_{p}^{+} \varepsilon_{p}^{-}-p^{2}+\left(\frac{\lambda^{2}-\Delta^{2}}{4}\right)+\lambda(\mathbf{p} \cdot \mathbf{k})\left\{\frac{\varepsilon_{k}^{-}-\varepsilon_{k}^{+}-\lambda}{k^{2}+E_{k, 1}^{+} E_{k, 1}^{-}}\right\}\right],
$$

with $\alpha=e^{2} / \epsilon \hbar v$ being the effective fine-structure constant that gives the strength of the interactions.

Using Eqs. (36) and (39), we write the expression for the total spin-flip transition moment as

$$
\mu+\delta \mu=\left\langle\downarrow\left|2 S_{x}\right| \uparrow\right\rangle\{1+\alpha F(2 k / \lambda, \Delta / \lambda)\} .
$$

To assess quantitatively the effect of the Coulomb interaction as a function of the band momentum, we plot the variation of the function $F(2 k / \lambda)$ with the rescaled momentum $(2 k / \lambda)$ for several values of $(\Delta / \lambda)$ in the bottom panel of Fig. 6 . The correction function is seen to be maximum at $k=0$ for all the different values of $(\Delta / \lambda)$ and is seen to decrease with increasing values of the band momenta. Although for $(\Delta / \lambda) \leqslant 1$ the bare transition moment $\mu$ was found to be zero at $k=0$, the Coulomb interaction correction effects turn out to be the largest for this regime. However, increasing the value of $(\Delta / \lambda)$ leads to a decrease in the Coulomb interaction correction. Of course, the correction function $F(2 k / \lambda)$ has to be multiplied by the dimensionless Coulomb interaction strength $\alpha \sim 1$, which is strongly material and environment dependent. It is clear from Fig. 6 that the overall interaction effect is strongest in the parameter regime $\Delta / \lambda \approx 0$, i.e., in the Kane-Mele universality class, while for $\Delta / \lambda>1$ and beyond the correction becomes gradually smaller and less pronounced even for substantial values of $\alpha$ as the system becomes dominated by the spin-independent gap.

\section{DISCUSSION AND OUTLOOK}

In summary we have analyzed the effect of Coulomb interactions on the spin transition magnetic moment for the case of atomically thin hexagonal lattices with spin-orbit interactions, such as 2D topological insulators (described by the Kane-Mele model), dielectric group-VI dichalcogenides, and the silicene-germanene class of materials. Due to the nonrelativistic nature of these systems and because of the two-dimensional nature of all the studied materials (meaning the spin-orbit interaction is a relatively small effect on top of the band structure), the anomalous, i.e., Coulomb, interaction effect manifests itself anisotropically and, indeed, only in the spin-flip channel for magnetic fields in the material planes. This is in contrast (although conceptually and technically very similar in spirit) to the famous anomalous magnetic moment of the electron in relativistic QED where the Schwinger result renormalizes directly and isotropically the electron $g$ factor. We can view our results as yet another important manifestation of (moderately strong) electron-electron interaction effects in graphenelike hexagonal monolayer systems which exhibit Dirac quasiparticle spectra.

As discussed in the previous section, which contains results across all parameter regimes (Fig. 6), it appears that the Kane-Mele limit (i.e., no spin-independent gap but a gap induced by the spin-orbit interaction) represents the point in parameter space where the Coulomb corrections are the strongest (Fig. 2). On the other hand, the monolayer dichalcogenides which are characterized by $\alpha$ as large as $\alpha \approx 4$ (much larger than suspended graphene with SOC) have relatively large gaps but reside firmly in the parameter regime $\Delta / \lambda>1$, making the anomalous effects much smaller and therefore harder to detect (see Fig. 4). We also note that our calculations were performed to first order in the bare Coulomb interaction $\alpha$ when the interaction effects were small, while we have used the RPA approximation, which takes into account self-consistent screening, for large bare $\alpha$ (relevant to suspended samples). The difference between the two approaches is important in practice only for the Kane-Mele model. Additionally, our work displays that the control of interactions can be achieved, for example, by using different substrates which can affect the Coulomb interaction via different levels of dielectric screening.

The anomalous spin contributions investigated in this work could lead to detectable signatures in experiments sensitive to spin relaxation and decoherence phenomena. For the case of sufficient spin-orbit coupling and band gaps in the range of $\sim 1 \mathrm{eV}$, a very promising magneto-optical Kerr effect technique previously employed to measure spin decoherence times $[39,40]$ may be sensitive enough to detect such anomalous contributions. However, it is important to emphasize that 
the spin relaxation mechanism in $2 \mathrm{D}$ materials is very material specific and depends strongly on various parameters such as ripples, phonons, the nature of substrates, and magnetic impurities [41-46]. It would be interesting to investigate the effect of anomalous spin contributions on the spin relaxation mechanism with the inclusion of various dissipative effects, such as phonons, ripples, and impurities. A microscopic theory that studies the effect of anomalous spin contributions on spin-flip lifetimes is well beyond the scope of the present work and is left for the future.

\section{ACKNOWLEDGMENTS}

S.S. is grateful to Prof. I. Garate for insightful discussion during the initial formulation of this work. S.S. was funded by the Canada First Research Excellence Fund. V.N.K. gratefully acknowledges the financial support of the Gordon Godfrey visitors program at the School of Physics, University of New South Wales, Sydney, during two research visits. V.N.K. also acknowledges financial support from NASA Grant No. 80NSSC19M0143.
[1] A. H. Castro Neto, F. Guinea, N. M. R. Peres, K. S. Novoselov, and A. K. Geim, Rev. Mod. Phys. 81, 109 (2009).

[2] M. I. Katsnelson, K. S. Novoselov, and A. K. Geim, Nat. Phys. 2, 620 (2006).

[3] M. Katsnelson and K. Novoselov, Solid State Commun. 143, 3 (2007).

[4] V. N. Kotov, B. Uchoa, V. M. Pereira, F. Guinea, and A. H. Castro Neto, Rev. Mod. Phys. 84, 1067 (2012).

[5] K. Novoselov, A. Geim, S. Morozov, D. Jiang, M. Katsnelson, I. Grigorieva, S. Dubonos, and A. Firsov, Nature (London) 438, 197 (2005).

[6] Y. Zhang, Y.-W. Tan, H. L. Stormer, and P. Kim, Nature (London) 438, 201 (2005).

[7] A. Chen, R. Ilan, F. de Juan, D. I. Pikulin, and M. Franz, Phys. Rev. Lett. 121, 036403 (2018).

[8] V. P. Gusynin and S. G. Sharapov, Phys. Rev. Lett. 95, 146801 (2005).

[9] Y. Zheng and T. Ando, Phys. Rev. B 65, 245420 (2002).

[10] M. Offidani and A. Ferreira, Phys. Rev. Lett. 121, 126802 (2018).

[11] S. Murakami, Phys. Rev. Lett. 97, 236805 (2006).

[12] C. L. Kane and E. J. Mele, Phys. Rev. Lett. 95, 226801 (2005).

[13] K. S. Novoselov, D. Jiang, F. Schedin, T. J. Booth, V. V. Khotkevich, S. V. Morozov, and A. K. Geim, Proc. Natl. Acad. Sci. USA. 102, 10451 (2005).

[14] D. Xiao, G.-B. Liu, W. Feng, X. Xu, and W. Yao, Phys. Rev. Lett. 108, 196802 (2012).

[15] S. Cahangirov, M. Topsakal, E. Aktürk, H. Şahin, and S. Ciraci, Phys. Rev. Lett. 102, 236804 (2009).

[16] P. Vogt, P. De Padova, C. Quaresima, J. Avila, E. Frantzeskakis, M. C. Asensio, A. Resta, B. Ealet, and G. Le Lay, Phys. Rev. Lett. 108, 155501 (2012).

[17] M. Z. Hasan and C. L. Kane, Rev. Mod. Phys. 82, 3045 (2010).

[18] C. L. Kane and E. J. Mele, Phys. Rev. Lett. 95, 146802 (2005).

[19] Y. Yao, F. Ye, X.-L. Qi, S.-C. Zhang, and Z. Fang, Phys. Rev. B 75, 041401(R) (2007).

[20] H. Min, J. E. Hill, N. A. Sinitsyn, B. R. Sahu, L. Kleinman, and A. H. MacDonald, Phys. Rev. B 74, 165310 (2006).

[21] V. B. Berestetskii, E. M. Lifshitz, and L. P. Pitaevskii, Quantum Electrodynamics, Landau and Lifshitz Vol. 4 (ButterworthHeinemann, Oxford, 1982).

[22] J. Schwinger, Phys. Rev. 73, 416 (1948).
[23] E. C. I. van der Wurff and H. T. C. Stoof, Phys. Rev. B 94, 155118 (2016).

[24] H. Hatami, T. Kernreiter, and U. Zülicke, Phys. Rev. B 90, 045412 (2014).

[25] M. Ezawa, Phys. Rev. B 86, 161407(R) (2012).

[26] C. J. Tabert, J. P. Carbotte, and E. J. Nicol, Phys. Rev. B 91, 035423 (2015).

[27] L. Stille, C. J. Tabert, and E. J. Nicol, Phys. Rev. B 86, 195405 (2012).

[28] C. J. Tabert and E. J. Nicol, Phys. Rev. Lett. 110, 197402 (2013).

[29] C. J. Tabert and E. J. Nicol, Phys. Rev. B 87, 235426 (2013).

[30] C. J. Tabert and E. J. Nicol, Phys. Rev. B 88, 085434 (2013).

[31] C. J. Tabert and E. J. Nicol, Phys. Rev. B 89, 195410 (2014).

[32] E. V. Gorbar, V. P. Gusynin, V. A. Miransky, and I. A. Shovkovy, Phys. Rev. B 66, 045108 (2002).

[33] V. N. Kotov, V. M. Pereira, and B. Uchoa, Phys. Rev. B 78, 075433 (2008).

[34] Z. Ni, Q. Liu, K. Tang, J. Zheng, J. Zhou, R. Qin, Z. Gao, D. Yu, and J. Lu, Nano Lett. 12, 113 (2012).

[35] M. Ezawa, Eur. Phys. J. B 85, 363 (2012).

[36] N. D. Drummond, V. Zólyomi, and V. I. Fal'ko, Phys. Rev. B 85, 075423 (2012).

[37] C.-C. Liu, H. Jiang, and Y. Yao, Phys. Rev. B 84, 195430 (2011).

[38] M. Ezawa, Phys. Rev. Lett. 109, 055502 (2012).

[39] L. Yang, N. A. Sinitsyn, W. Chen, J. Yuan, J. Zhang, J. Lou, and S. A. Crooker, Nat. Phys. 11, 830 (2015).

[40] M. Furis, D. L. Smith, S. Kos, E. S. Garlid, K. S. M. Reddy, C. J. Palmstrøm, P. A. Crowell, and S. A. Crooker, New J. Phys. 9, 347 (2007).

[41] D. Huertas-Hernando, F. Guinea, and A. Brataas, Phys. Rev. Lett. 103, 146801 (2009).

[42] C. Ertler, S. Konschuh, M. Gmitra, and J. Fabian, Phys. Rev. B 80, 041405(R) (2009).

[43] S. Fratini, D. Gosálbez-Martínez, P. Merodio Cámara, and J. Fernández-Rossier, Phys. Rev. B 88, 115426 (2013).

[44] M. B. Lundeberg, R. Yang, J. Renard, and J. A. Folk, Phys. Rev. Lett. 110, 156601 (2013).

[45] D. V. Tuan, F. Ortmann, D. Soriano, S. O. Valenzuela, and S. Roche, Nat. Phys. 10, 857 (2014).

[46] A. H. Castro Neto and F. Guinea, Phys. Rev. Lett. 103, 026804 (2009). 